\title{
Inducible co-stimulator inhibits lipid phagocytosis of human aortic smooth muscle cells by down-regulating CD36 expression
}

\author{
Meng Liang ${ }^{1,2}$, Xuemin $\mathrm{Guo}^{3}$, Heming $\mathrm{Wu}^{3} \wedge$, Zhixiong Zhong ${ }^{2,3}$ \\ ${ }^{1}$ The First Clinical Medical College, Gannan Medical University, Ganzhou, China; ${ }^{2}$ Center for Cardiovascular Diseases, Meizhou People's Hospital \\ (Huangtang Hospital), Meizhou Academy of Medical Sciences, Meizhou, China; ${ }^{3}$ Guangdong Provincial Key Laboratory of Precision Medicine and \\ Clinical Translational Research of Hakka Population, Meizhou People's Hospital (Huangtang Hospital), Meizhou Academy of Medical Sciences, \\ Meizhou, China \\ Contributions: (I) Conception and design: Z Zhong, X Guo; (II) Administrative support: Z Zhong; (III) Provision of study materials or patients: Z \\ Zhong; (IV) Collection and assembly of data: M Liang; (V) Data analysis and interpretation: M Liang, X Guo, H Wu; (VI) Manuscript writing: All \\ authors; (VII) Final approval of manuscript: All authors. \\ Correspondence to: Prof. Zhixiong Zhong. Center for Cardiovascular Diseases, Center for Precision Medicine, Guangdong Provincial Key Laboratory \\ of Precision Medicine and Clinical Translational Research of Hakka Population, Meizhou People's Hospital (Huangtang Hospital), Meizhou \\ Academy of Medical Sciences, No. 63 Huangtang Road, Meijiang District, Meizhou 514031, China. Email: zhongzhixiong@mzrmyy.com.
}

Background: To explore the potential mechanism of inducible co-stimulator (ICOS) inhibition of lipid phagocytosis in human aortic smooth muscle cells (HASMCs).

Methods: Excess Dil (1,1'-dioctadecyl-3,3,3',3'-tetramethylindocarbocyanine perchlorate)-labeled oxidized low-density lipoprotein (ox-LDL) was used to induce HASMCs to form a foam cell model; HASMCs were cultured together with ICOS-overexpressed JurKat (JK-ICOS) cells or recombinant human ICOS protein (rICOS protein) to be stimulated, and a confocal laser microscope was used to observe the lipid phagocytosis of HASMCs. Reverse transcription quantitative polymerase chain reaction (RT-qPCR), western blot, and immunofluorescence staining were used to detect the expression of the lipid phagocytic receptor, cluster of differentiation 36 (CD36) in HASMCs.

Results: The uptake of Dil ox-LDL by HASMCs was concentration-dependent, and excessive Dil oxLDL uptake led to lipid accumulation in HASMCs. Pretreatment with JK-ICOS cells or rICOS protein for HASMCs 48 hours reduced Dil ox-LDL-induced lipid accumulation. Compared with HASMCs cocultured with empty lentiviral JurKat (JK-EV) cells, the messenger RNA (mRNA) and protein expressions of CD36 in HASMCs co-cultured with JK-ICOS cells were significantly down-regulated. The results of immunofluorescence staining showed that co-culturing with JK-ICOS cells could down-regulate ox-LDLinduced expression of CD36 in HASMCs, but JK-EV cells could not. Similarly, the results of qPCR, western blot, and immunofluorescence staining showed that rICOS protein could down-regulate the ox-LDLinduced expression of CD36 in HASMCs, but this down-regulation was not as significant as that in JK-ICOS cells.

Conclusions: ICOS could inhibit the lipid phagocytosis of HASMCs by down-regulating the expression of CD36, suggesting a potential anti-atherosclerosis (anti-AS) mechanism of ICOS, and preventing ox-LDLinduced formation of myogenic foam cells.

Keywords: Inducible co-stimulator (ICOS); lipid phagocytosis; atherosclerosis (AS); lipid phagocytic CD36; lowdensity lipoprotein

Submitted Nov 19, 2021. Accepted for publication Jan 07, 2022.

doi: $10.21037 /$ jtd-21-1901

View this article at: https://dx.doi.org/10.21037/jtd-21-1901

^ ORCID: 0000-0002-1876-9585. 


\section{Introduction}

Atherosclerosis (AS) is a chronic lipid-induced inflammation of the vascular wall with complex etiology, which can lead to cardiovascular disease (CVD) (1). The accumulation of low-density lipoprotein (LDL) and oxidation modification causing focal fibroinflammatory degeneration of the arterial intima are the main characteristics of AS (2). In particular, oxidized-LDL (ox-LDL) is considered the main factor of AS, which can be recognized and captured by the scavenger receptors in tissues. Studies have shown that oxLDL can cause inflammation, proliferation, and excessive production of reactive oxygen species in cardiovascular cells $(3,4)$. Monocytes/macrophages, vascular smooth muscle cells, and platelets have also been shown to express specific scavenger receptors for ox-LDL $(5,6)$. Vascular smooth muscle cells (VSMC) are mainly located in the media of the aorta. In the early lesions of AS, LDL is oxidized through damaged endothelial cells to make ox-LDL, ox-LDL can be recognized and phagocytosed by VSMC in addition to macrophages, thus forming muscle-derived foam cells, which constitute the main components of lipid stripes in early atherosclerotic lesions. After that, the function of foam cells is destroyed, resulting in cell necrosis and disintegration, which eventually leads to accumulation of atherosclerotic necrotic material and promotes the formation of AS (7). In addition, during the development of AS, VSMC proliferate and migrate from the media to the lesions, covering the early lipid plaques to form fibrous plaques, resulting in intimal thickening and arterial lumen stenosis, which eventually lead to AS (8). It was worthy to study of VSMC, which as an important source of foam cells in $\mathrm{AS}$.

Cluster of differentiation 36 (CD36) is a multifunctional single-chain transmembrane protein with a relative molecular mass of 78-88 $\mathrm{KD}$ in the $\mathrm{B}$-type scavenger receptor family, which is expressed in monocytes/macrophages, smooth muscle cells, and cardiomyocytes (9-11). Studies have shown that the uptake of ox-LDL after peripheral blood mononuclear cells infiltrate the arterial wall can lead to the expression of CD36 and promote the formation of foam cells (12). High concentrations of glucose can stimulate the lipotoxicity, apoptosis, and fibrosis of human kidney cells induced by oxidized protein, by up-regulating the expression of CD36 receptors (13). Overexpression of CD36 on human umbilical artery smooth muscle cells and human leukemic monocyte cells can promote lipid aggregation caused by ox-LDL (14).
Free fatty acids released by lipid metabolism in catalase $\mathrm{KO}$ mice are transported to brown adipose tissue through the activation of CD36, and then degraded by lipoprotein lipase (15). During the culture of rainbow trout primary adipocytes, the expression of CD36 gradually increased with the differentiation and maturation of primary adipocytes (16). These research results strongly illustrate the importance of CD36 to lipid uptake and metabolism, and foam cell formation.

Inducible co-stimulator (ICOS), also known as CD278, is a member of the CD28 family. After being stimulated by antigens, ICOS is expressed on activated $\mathrm{T}$ lymphocytes to enhance the immune response of T lymphocytes to antigens (17). Loss of ICOS signaling can promote the accumulation of regulatory $T$ cells in visceral adipose tissue (18). A previous study found a large number of ICOS expressions in atherosclerotic plaques of $\mathrm{ApoE}^{-/-}$mice, and proposed the possibility that ICOS has a protective effect on AS (19). However, this was not explicitly verified in the study, and its mechanism was not explored in depth. Our preliminary experiments have found that the expression of ICOS in the aortic atherosclerotic plaques of APOE KO rats was significantly reduced, and the overexpression of ICOS could inhibit the phagocytosis and proliferation of HASMCs (20). This experiment tested the main lipid phagocytic receptors involved and found CD36 has significant differences. A large number of studies have shown that CD36 is one of the main phagocytic receptors of ox-LDL (12,21-24), but there is no literature report on the exact relationship between ICOS and CD36. Therefore, the present study takes this as a starting point to conduct an in-depth study on the correlation between the lipid phagocytosis of HASMCs and ICOS. This study is the first time to study the interaction between ICOS and lipid phagocytic receptors CD36 on HASMCs, and will explore the possible role of ICOS in the lipid phagocytic function of HASMCs and the potential antiAS mechanism. We present the following article in accordance with the MDAR reporting checklist (available at https://jtd. amegroups.com/article/view/10.21037/jtd-21-1901/rc).

\section{Methods}

\section{Cell line and cell culture and grouping}

A stable human T lymphocyte leukemia cell line (JurKat T cell) with ICOS overexpression was constructed using the lentivirus packaging technique (20). HASMCs were derived from the American Type Culture Collection (ATCC) cell 


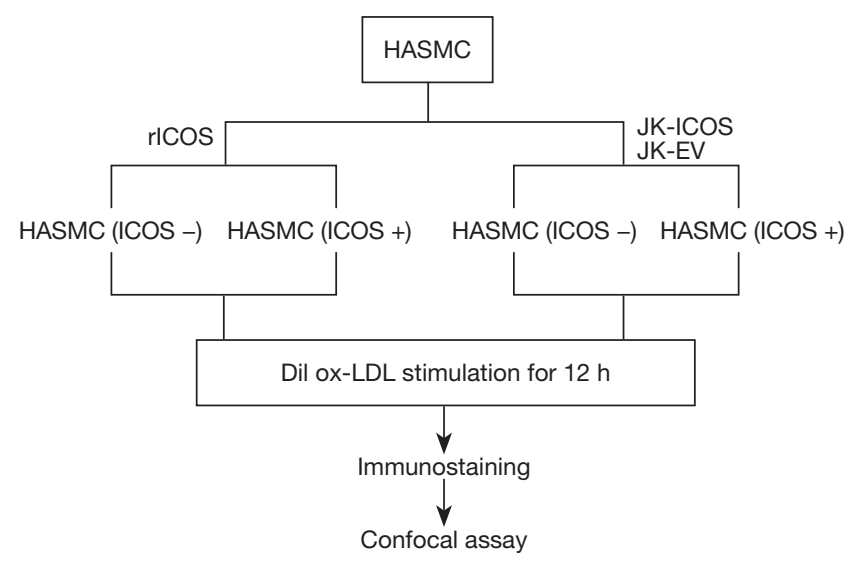

Figure 1 Flow chart of Dil ox-LDL uptake by HASMCs. HASMC, human aortic smooth muscle cell; rICOS, recombinant human ICOS protein; JK-ICOS, ICOS-overexpressed JurKat cells; JK-EV, empty lentiviral JurKat cells; Dil ox-LDL, Dillabeled oxidized low-density lipoprotein; Immunostaining, immunofluorescence staining; ICOS, inducible co-stimulator.

bank and cultured in a complete medium of HASMCs (CHI Scientific, China, Cat. No. 3-0202) [Dulbecco's Modified Eagle's medium (DMEM) supplemented with $10 \%$ containing fetal bovine serum (FBS), $100 \mathrm{U} / \mathrm{mL}$ penicillin, and $100 \mathrm{U} / \mathrm{mL}$ streptomycin]. JurKat $\mathrm{T}$ cells were cultured with RPMI 1640 medium (Gibco, America, Cat. No. C11875500BT) containing 10\% FBS (Scitecher, Australia, Cat. No. AU-FBS-500) and 1\% penicillin /streptomycin (Solarbio, China, Cat. No. P1400) in a T75 cell culture bottle.

There were two groups: (I) JK-ICOS cells and HASMCs co-culture group with ox-LDL stimulation (ox-LDL+JKICOS); (II) no-load lentiviral JurKat T (JK-EV) cells and HASMCs co-culture group with ox-LDL stimulation (oxLDL+JK-EV). Cells $\left(5 \times 10^{4}\right)$ of HASMC suspension were placed in a $6 \mathrm{~cm}$ cell culture dish, and after 24 hours, the old medium was discarded, washed with PBS (Solarbio, China, Cat. No. P1020), replaced with fresh medium, and JK-ICOS cells or JK-EV cells were added to co-culture. After 24 hours, $50 \mu \mathrm{g} / \mathrm{mL}$ ox-LDL (Yiyuan Biotechnology, China, Cat. No. YB-002) was added for 48 hours.

\section{Dil-labeled ox-LDL uptake assay}

The cell slide was placed in a 24-well plate and the HASMC suspension was added. After the cells adhered, they were stimulated with JurKat $\mathrm{T}$ cells or recombinant human ICOS protein (Sino Biological, China, Cat. No. 10344-H31H) for 48 hours. Ten $\mu \mathrm{g} / \mathrm{mL}$ fluorescently-labeled oxidized lowdensity lipoprotein (Dil ox-LDL) (Invitrogen, America Cat. No. L34358) was added in the dark, and the 24-well plate was wrapped in tin foil to continue culturing for 12 hours (Figure 1). The subsequent steps were all performed under dark conditions: the cells were washed with PBS 3-4 times, the cell slide was taken out and covered on a glass slide dripped with anti-fluorescence attenuation sealing liquid containing DAPI (Solarbio, China, Cat. No. S2110), and finally fixed. We observed and analyzed the lipid uptake of HASMCs under a laser confocal microscope.

\section{$R N A$ isolation and reverse transcription quantitative polymerase chain reaction ( $R T-q P C R)$}

Total cellular RNA was extracted using an RNA simple total RNA extraction kit (TIANGEN, China, Cat. No. DP419) according to the manufacturer's instructions, and the concentration was measured. Reverse transcription of $500 \mathrm{ng}$ total RNA was performed using Prime Script ${ }^{\mathrm{TM}}$ RT Master Mix (Perfect Real Time) (TaKaRa, Japan, Cat. No. RR036B). RT-qPCR was performed using TB Green Premix Ex Taq ${ }^{\text {TM }}$ II (Tli RNaseH Plus) (TaKaRa, Japan, Cat. No. RRB20A) kit and applied Biosystems 7500 Fast Real-Time PCR System (Thermo Fisher Scientific, America), with the following primers: for CD36, 5'-CCAGTTGGAGACCTGCTTATC-3' and 5'-TCTGTAAACTTCTGTGCCTGTT-3'; and for glyceraldehyde-3-phosphate dehydrogenase (GAPDH), 5'-ATGACATCAAGAAGGTGGTG-3' and 5'-CATACCAGGAAATGAGCTTG-3'. The relative foldchange was determined using the double delta method $\left(2^{-\Delta \Delta C T}\right)$ normalized to GAPDH level and data analysis was performed using Prism7 software (GraphPad Software, Inc., California, USA).

\section{Western blot analysis}

Cellular total proteins were extracted and the concentration of total protein was detected using the bicinchoninic acid (BCA) (Beyotime, China, Cat. No. P0012) method. An equivalent amount of total protein was added to $10 \%$ sodium dodecyl sulfate-polyacrylamide gel electrophoresis (SDS-PAGE) (Solarbio, China, Cat. No. P1200) and transferred to a polyvinylidene fluoride (PVDF) (Merck millipore, Germany, Cat. No. IPVH00010) membrane via 
a slot transfer blotting system. The PVDF membrane was blocked with $5 \%$ bovine serum albumin (BSA) (SigmaAldrich, America, Cat. No. B2064-100G) prepared by $1 \times$ tris buffered saline with tween (TBST) (Sigma-Aldrich, America, Cat. No. P7949-500ML), and blocked on a shaker at room temperature for 2 hours. The blot was incubated with anti-CD36 (1:2,000) (Abcam, UK, Cat. No. ab133625) and anti-GAPDH $(1: 5,000)$ (Abcam, UK, Cat. No. ab181602) antibodies at $4{ }^{\circ} \mathrm{C}$ overnight. After washing with $1 \times$ TBST three times, the blot was incubated with anti-rabbit secondary antibody $(1: 10,000)$ (Abcam, UK, Cat. No. ab6721) on a shaker at room temperature for $2 \mathrm{~h}$. After washing with $1 \times$ TBST three times, a chemiluminescence system was used to detect immunoreactive bands.

\section{Immunofuorescence staining assay}

The cell slide was placed in a 24-well plate, and HASMC suspension was added. After cell adhesion, JurKat T cells or rICOS protein were added to co-culture for 24 hours, and $50 \mu \mathrm{g} / \mathrm{mL}$ ox-LDL was added to culture, for 48 hours. The cell slide with adhered cells was washed in the 24-well cell culture plate with PBS three times (3 minutes each time), and then fixed with $4 \%$ tissue cell fixative (Solarbio, China, Cat. No. P1110) for 15 minutes. After washing with PBS three times, 5\% BSA was added to block for 30 minutes. The cell slide was taken out and placed on a glass slide. An immunohistochemical PAP pen (Solarbio, China, Cat. No. YA0310) was used to draw a circle of appropriate size on the cell slide, and the 1:50 diluted anti-CD36 recombinant rabbit monoclonal antibody (Huabio, China, Cat. No. ET1701-24) was dropped into the circle. The cell slides were placed in a wet box and then in a refrigerator at $4{ }^{\circ} \mathrm{C}$ overnight. On the second day, put the cell slide back into the cell culture plate and washed with phosphate buffered saline (PBS) containing $0.05 \%$ Tween 20 (PBST) three times. Next, the 1:1,000 diluted iFluor ${ }^{\mathrm{TM}} 488$ Goat antirabbit IgG antibody (Huabio, China, Cat. No. HA1121) was added in the dark, and the cell culture plate was wrapped with tin foil and placed on a shaker to incubate for 1 hour at room temperature. The subsequent steps were all performed under dark conditions: the cell slide was washed with PBST three times, and subsequently taken out and cover it on a glass slide with anti-fluorescence attenuation sealing liquid containing DAPI. The lipid uptake of HASMCs was observed and analyzed under a laser confocal microscope.

\section{Statistical analysis}

Data were presented as the mean \pm standard error (SE). The difference between the two groups was determined by unpaired two-tailed Student's $t$-test. A P value $<0.05$ was considered statistically significant. All statistical analyses were performed with the GraphPad Prism 7.0 software.

\section{Results}

\section{HASMCs lipid phagocytosis significantly inbibited after treatment with ICOS conferred by $7 \mathrm{~K}$-ICOS cells}

Foam cells formed by HASMCs after phagocytosis of lipids are one of the reasons for the formation of atherosclerotic plaques. In this study, we first detected the relationship between ox-LDL and HASMCs. Dil ox-LDL at concentrations of 5,10 , and $30 \mu \mathrm{g} / \mathrm{mL}$ were stimulated to HASMCs for 12 hours, and the phagocytosis of Dil oxLDL in HASMCs was observed under a laser confocal microscope. It could be seen that compared with the control group, the amount of Dil ox-LDL phagocytosis in HASMCs increased significantly with the increase of Dil ox-LDL concentration, and when the concentration of Dil ox-LDL was $10 \mu \mathrm{g} / \mathrm{mL}$, the amount of phagocytosis of Dil ox-LDL in HASMCs was prominent (Figure $2 A$ ). However, there was no significant difference in the content of Dil ox-LDL in HASMCs between the two groups with concentrations of 10 and $30 \mu \mathrm{g} / \mathrm{mL}$ (Figure $2 A$ ). The results indicated that the lipid content in HASMCs is Dil ox-LDL concentration-dependent, and when the concentration reaches a certain amount, the phagocytosis of Dil oxLDL by HASMCs will stop. When the concentration of Dil ox-LDL was $10 \mu \mathrm{g} / \mathrm{mL}$, the lipid phagocytosis effect of HASMCs was already significant, and therefore, this concentration was to stimulate HASMCs in subsequent fluorescence phagocytosis experiments.

It has been reported that ICOS may play an antiatherosclerotic role by inhibiting the phagocytosis and proliferation of HASMCs during the formation of atherosclerotic plaques (20). In this experiment, the relationship between ICOS and HASMCs was detected by co-cultivation of JK-ICOS cells and HASMCs. JK-ICOS cells and JK-EV cells were co-cultured with HASMCs at a ratio of 1:5, 2:5, and 5:5 for 48 hours, respectively. Dil ox-LDL was then added and stimulated for 12 hours, and the phagocytosis of Dil ox-LDL in HASMCs was observed under a laser confocal microscope. The results 
A

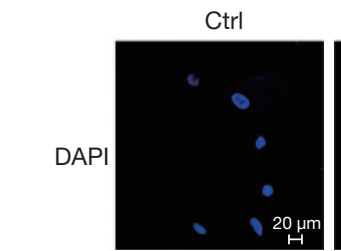

$5 \mu \mathrm{g} / \mathrm{mL}$

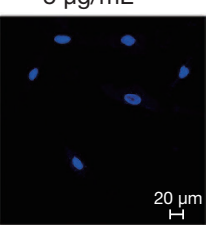

$10 \mu \mathrm{g} / \mathrm{mL}$

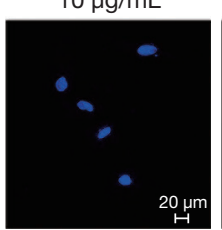

$30 \mu \mathrm{g} / \mathrm{mL}$
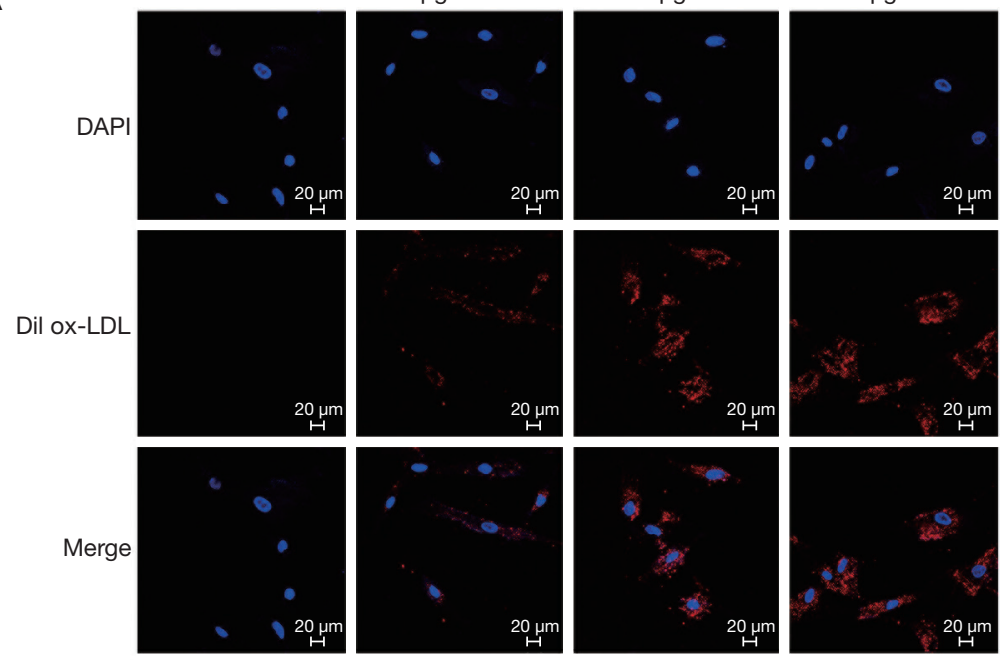

DIC

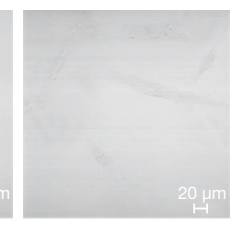

B

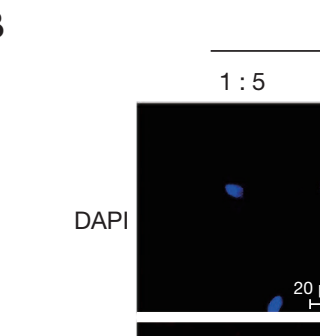
JurKat-EV: HASMC
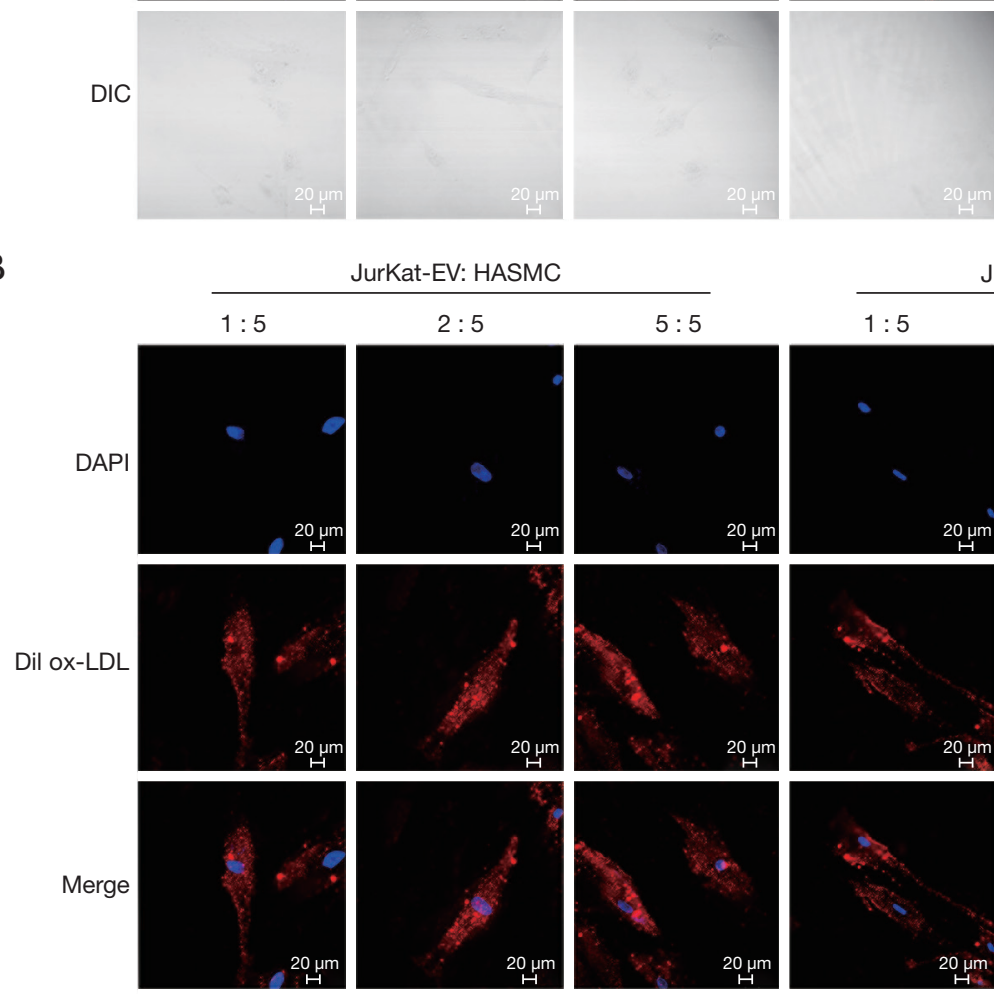

$5: 5$
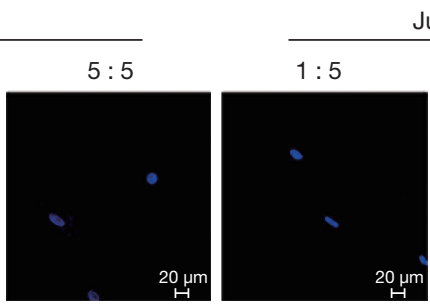

JurKat-ICOS: HASMC
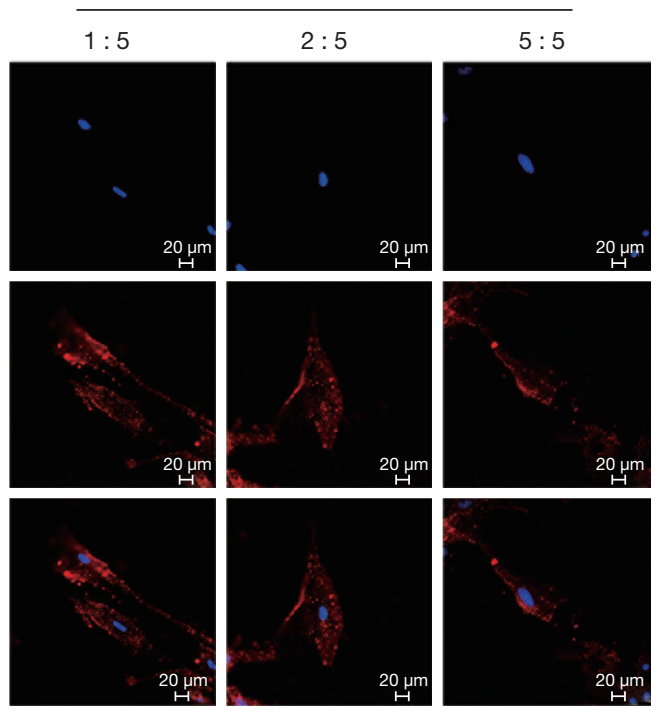

DIC

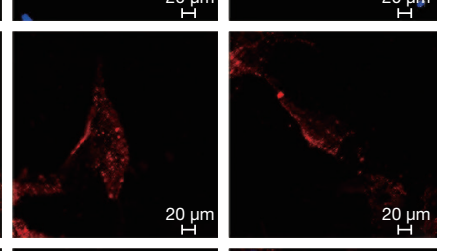

Figure 2 Effect of ICOS conferred by JK-ICOS cells on lipid phagocytosis in HASMCs. (A) The uptake of Dil ox-LDL in HASMCs after treating HASMCs with different concentrations of Dil ox-LDL for 12 hours using a laser confocal microscope. Scale bars represent $20 \mu \mathrm{m}$; (B) The uptake of Dil ox-LDL in HASMCs after 48 hours of co-culture with JK-ICOS/JK-EV cells in different proportions using a laser confocal microscope. Scale bars represent $20 \mu \mathrm{m}$. Immunofluorescence staining: DAPI, cell nucleus stained blue; Dil ox-LDL, Dil-labeled ox-LDL stained red; Merge, merge these two pictures; DIC, cell morphology photographed in bright field. JK-EV, empty lentiviral JurKat; JK-ICOS, ICOS-overexpressed JurKat; Dil ox-LDL, Dil-labeled oxidized low-density lipoprotein; HASMC, human aortic smooth muscle cell. 

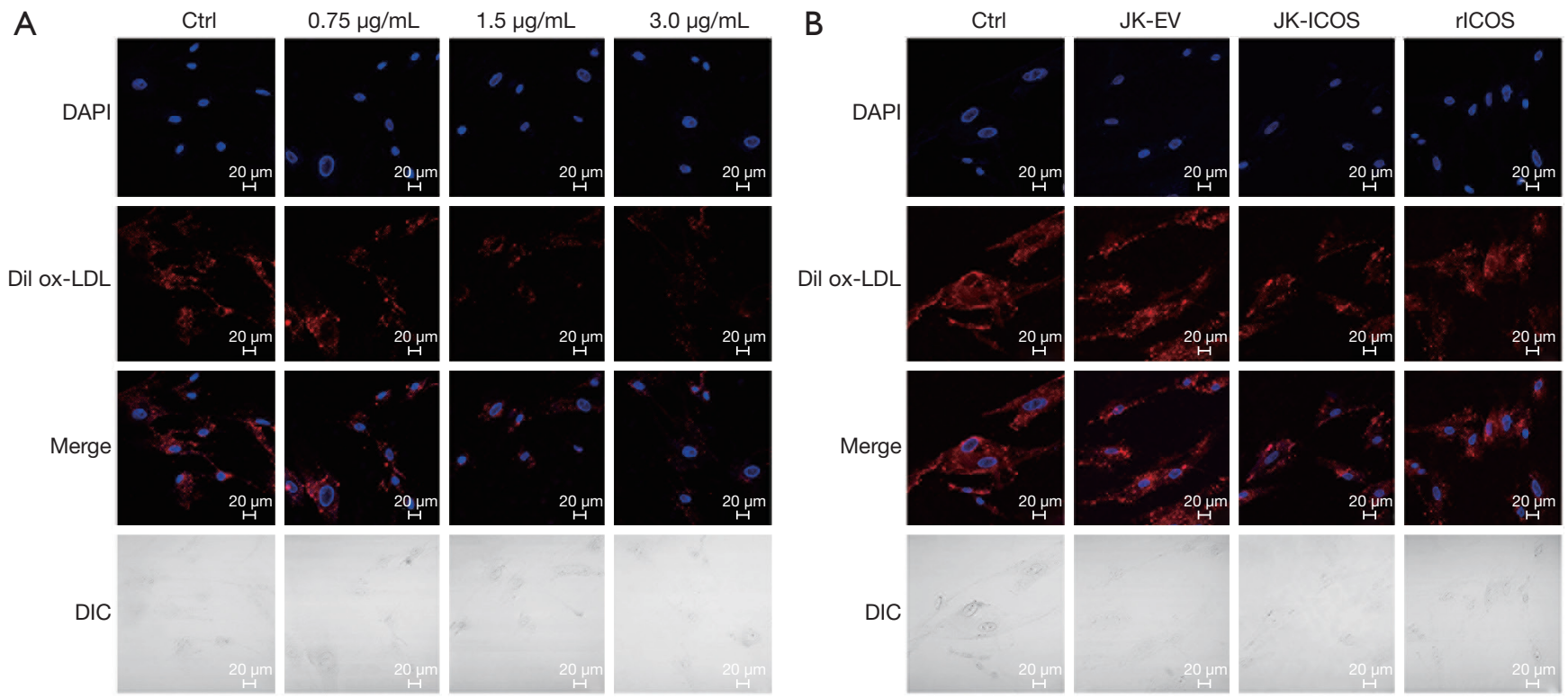

Figure 3 Effect of rICOS protein on lipid phagocytosis in HASMCs. (A)The uptake of Dilox-LDL in HASMCs stimulated by different concentrations of rICOS protein for 48 hours using a laser confocal microscope. Scale bars represent $20 \mu \mathrm{m}$. (B) The uptake of Dil oxLDL in HASMCs stimulated by JK-ICOS, JK-EV, and rICOS protein for 48 hours using a laser confocal microscope. Scale bars represent $20 \mu \mathrm{m}$. Immunofluorescence staining: DAPI, cell nucleus stained blue; Dil ox-LDL, Dil-labeled ox-LDL stained red; Merge, merge these two pictures; DIC, cell morphology photographed in bright field. JK-EV, empty lentiviral JurKat; JK-ICOS, ICOS-overexpressed JurKat; rICOS, recombinant human ICOS protein; ICOS, inducible co-stimulator; Dil ox-LDL, Dil-labeled oxidized low-density lipoprotein; HASMC, human aortic smooth muscle cell.

showed that in HASMCs incubated with JK-ICOS cells, the phagocytosis of Dil ox-LDL decreased as the proportion of JK-ICOS cells increased, and when the ratio of the two kinds of cells was 5:5, the amount of Dil oxLDL phagocytosed by HASMCs was lowest (Figure 2B). However, there was no significant difference in the phagocytosis of Dil ox-LDL among HASMCs incubated with different ratios of JK-EV cells (Figure 2B). At the same proportion, the JK-ICOS cells co-culture group had less phagocytosis of Dil ox-LDL in HASMCs compared with the JK-EV cells co-culture group (Figure $2 B$ ). These results suggested that JK-ICOS cells might inhibit HASMCs lipid phagocytosis after stimulating HASMCs.

\section{HASMCs lipid phagocytosis moderately inhibited after treatment with rICOS protein}

In order to further determine whether the inhibition of HASMCs lipid phagocytosis by JK-ICOS cells is due to ICOS, rICOS protein stimulated to HASMCs. HASMCs were stimulated with the rICOS protein at concentrations of $0.75,1.5$, and $3.0 \mu \mathrm{g} / \mathrm{mL}$, respectively, for 48 hours, and then Dil ox-LDL was added to stimulate the HASMCs for 12 hours. The result (under the microscope) showed that the intake of Dil ox-LDL in HASMCs decreased with the increase of ICOS protein concentration. Compared with the treatment group without the rICOS protein, when the concentration of rICOS protein was 1.5 and $3 \mu \mathrm{g} / \mathrm{mL}$, the Dil ox-LDL in HASMCs decreased significantly, but there was no marked difference between the two concentrations (Figure 3A).

By using JK-ICOS cells and the rICOS protein to stimulate HASMCs separately, it could be seen that compared with only the Dil ox-LDL treatment group, the Dil ox-LDL content in HASMCs stimulated by JK-ICOS cells or rICOS protein was reduced. And, the content in HASMCs after stimulation by JK-ICOS cells was more reduced (Figure 3B). These findings indicated that the ICOS gene may play an important role in inhibiting the lipid phagocytosis of HASMCs, but the inhibitory effect of ICOS alone is not as strong as the inhibitory effect of high-ICOSexpressing $\mathrm{T}$ cells. 
A

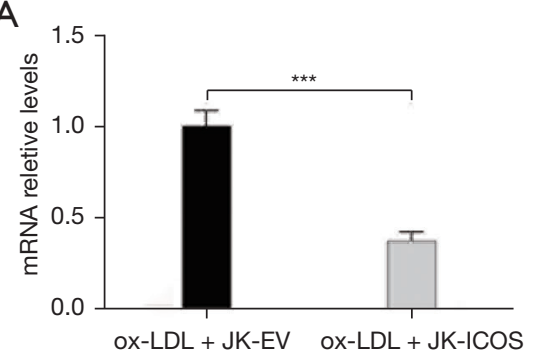

B

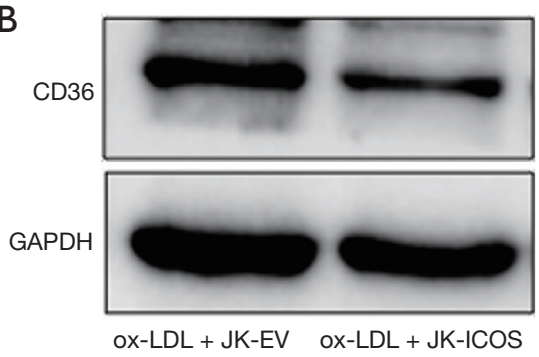

C
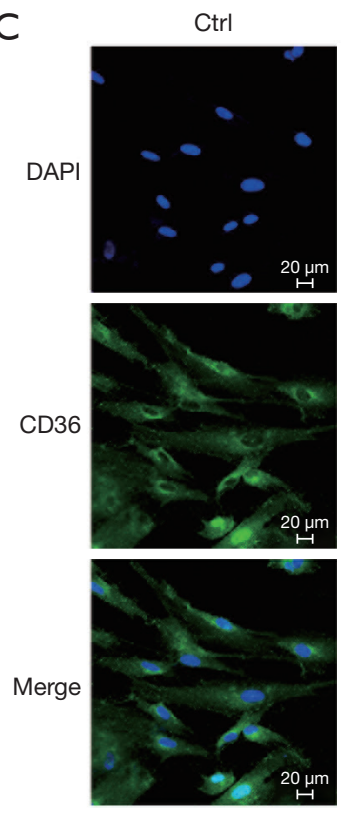

ox-LDL
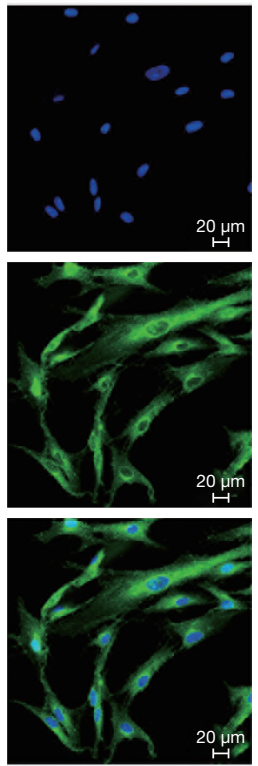

ox-LDL + JK-EV
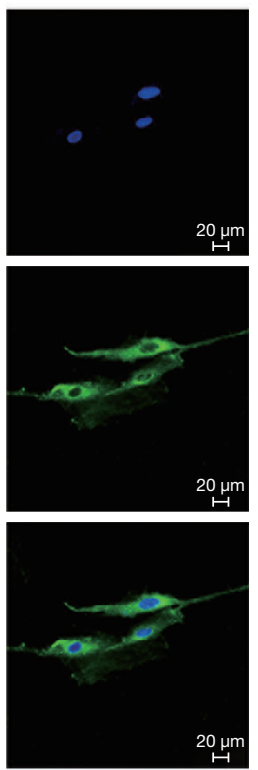

OX-LDL + JK-ICOS
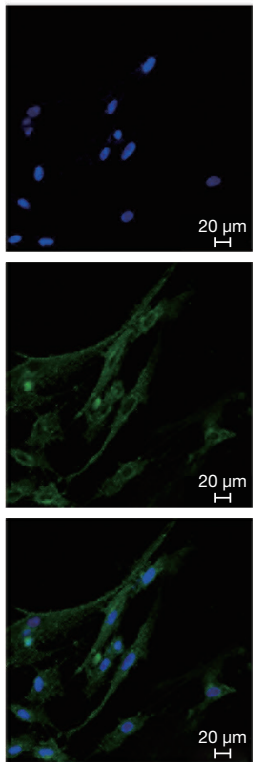

Figure 4 Effect of ICOS conferred by JK-ICOS cells on the expression of lipid phagocytic receptor CD36 in HASMCs. (A) RT-qPCR assays of the expression of CD36 mRNAs in HASMCs treated with ox-LDL and JK-EV/JK-ICOS cells for 48 hours. $\left.{ }^{* * *}, \mathrm{P}<0.001\right)$; (B) Western blot assays of the expression of CD36 mRNAs in HASMCs treated with ox-LDL and JK-EV/JK-ICOS cells for 48 hours; (C) observation of CD36 expression in HASMCs using a laser confocal microscope. Ctrl group: HASMCs without any treatment; ox-LDL group: ox-LDL stimulates HASMCs; ox-LDL + JK-EV group: ox-LDL and JK-EV cells stimulates HASMCs; ox-LDL+JK-ICOS group: ox-LDL and JK-ICOS cells stimulates HASMCs. Scale bars represent $20 \mu \mathrm{m}$. JurKat Immunofluorescence staining: DAPI, cell nucleus stained blue; Dil ox-LDL, Dil-labeled ox-LDL stained red; Merge, merge these two pictures; DIC, cell morphology photographed in bright field. RT-qPCR, reverse transcription quantitative PCR; WB, western blotting; CD36, cluster of differentiation 36; HASMC, human aortic smooth muscle cell; Ox-LDL, oxidized low-density lipoprotein; JK-EV, empty lentiviral JurKat; JK-ICOS, ICOS-overexpressed.

\section{Expression of lipid phagocytic receptor CD36 significantly down-regulated in HASMCs upon $7 \mathrm{~K}$-ICOS cells stimulation}

To delineate the underlying mechanisms through which ICOS regulates foam cell formation, the expression of CD36, one of the main phagocytic receptors of ox-LDL, was assessed. In this study, RT-qPCR, western blot, and immunofluorescence staining were performed to test the changes in the expression of CD36 on HASMCs treated with ox-LDL and stimulated by JK-ICOS cells or JKEV cells for 48 hours. In the case of ox-LDL stimulation, compared with HASMCs stimulated by JK-EV cells, the mRNA and protein expression levels of CD36 on HASMCs stimulated by JK-ICOS cells were significantly reduced (Figure $4 A, 4 B$ ). According to the immunofluorescence staining results, compared with the non-ox-LDL treatment group, the expression of CD36 on HASMCs treated with ox-LDL increased, whereas the expression of CD36 on
HASMCs stimulated by ox-LDL and JK-ICOS cells decreased significantly (Figure 4C). These experiments have shown that JK-ICOS cells may be able to inhibit the phagocytic function of HASMCs by reducing the expression of lipid phagocytic receptors. It is worth noting that, compared with only the ox-LDL treatment group, the CD36 expression in HASMCs stimulated by ox-LDL and JKEV cells did not change significantly, but was markedly lower in the ox-LDL and JK-ICOS cells stimulation groups (Figure 4C). This result suggested that JK-EV cells cannot down-regulate the increase in CD36 expression caused by ox-LDL, but JKICOS cells can, which further indicates that ICOS may play a vital role in down-regulating CD36 expression.

\section{Expression of CD36 moderately down-regulated in HASMCs upon rICOS protein stimulation}

At the same time, in order to investigate the role of 
A

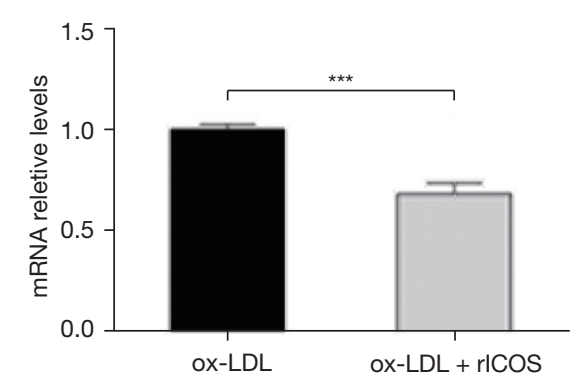

B

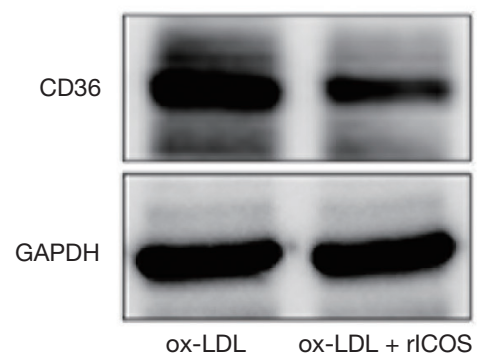

C
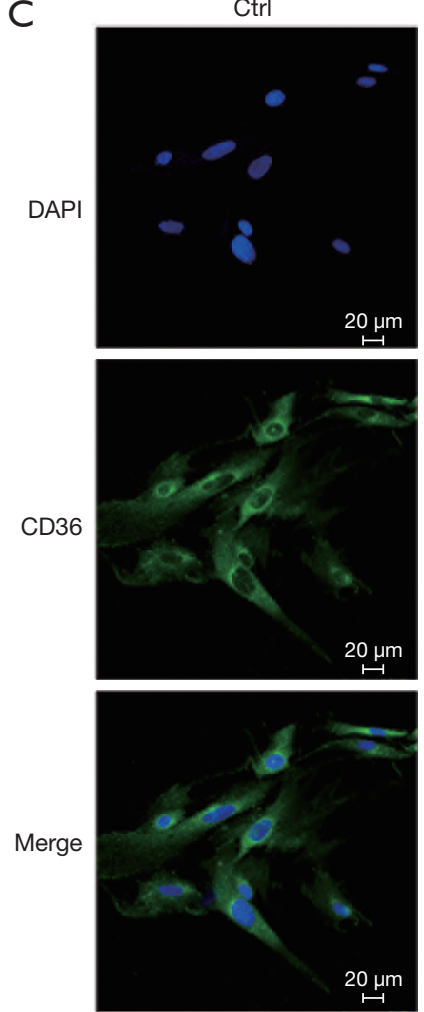

ox-LDL
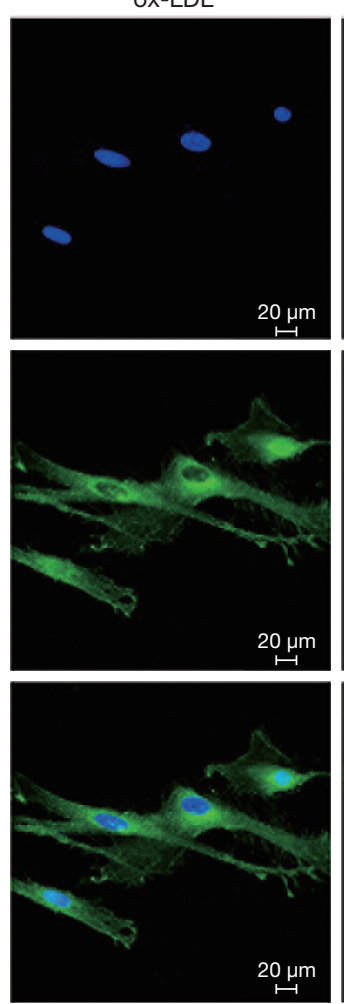

ox-LDL + rICOS
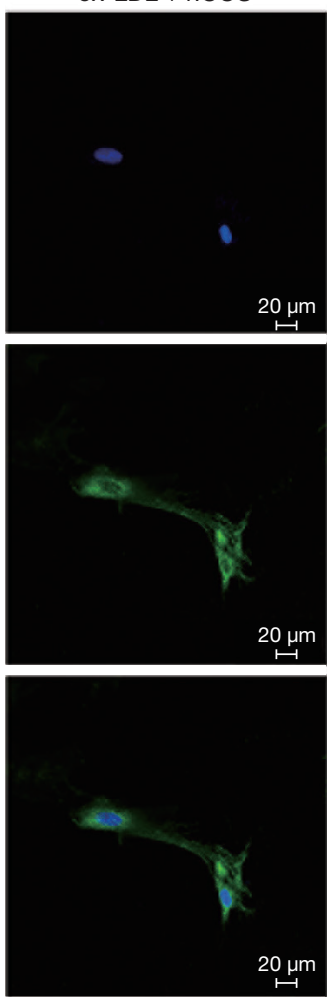

Figure 5 Effect of rICOS protein on the expression of CD36 in HASMCs. (A) RT-qPCR assays of the expression of CD36 mRNAs in HASMCs treated with ox-LDL and with/without rICOS protein for 48 hours. ${ }^{* * *}, \mathrm{P}<0.001$ ); (B) Western blot assays of the expression of CD36 mRNAs in HASMCs treated with ox-LDL and with/without rICOS protein for 48 hours; (C) observation of CD36 expression in HASMCs using a laser confocal microscope. Ctrl group: HASMCs without any treatment, ox-LDL group: ox-LDL stimulates HASMCs, ox-LDL + rICOS group: ox-LDL and rICOS protein stimulates HASMC. Scale bars represent $20 \mu \mathrm{m}$. Immunofluorescence staining: DAPI, cell nucleus stained blue; Dil ox-LDL, Dil-labeled ox-LDL stained red; Merge, merge these two pictures; DIC, cell morphology photographed in bright field. RT-qPCR, reverse transcription quantitative PCR; WB, western blotting; CD36, cluster of differentiation 36; HASMC, human aortic smooth muscle cell; ox-LDL, oxidized low-density lipoprotein; rICOS, recombinant human ICOS protein.

the ICOS gene, the influence of rICOS protein on the expression of lipid phagocytic receptor CD36 in HASMCs was tested. Compared with the ox-LDL treatment group, the mRNA and protein expression of CD36 in HASMCs treated with ox-LDL and ICOS protein decreased (Figure 5A,5B). Immunofluorescence staining experiments showed that the expression of CD36 in HASMCs treated with ox-LDL was significantly higher than that in HASMCs without any treatment (Figure 5C). However, compared with HASMCs treated with ox-LDL, the expression of CD36 was decreased in HASMCs treated with ox-LDL and ICOS protein (Figure 5C). These results suggest that rICOS protein can inhibit the expression of CD36 in HASMCs.

\section{Discussion}

In this study, we used JK-ICOS cells/rICOS protein to stimulate HASMCs, and observed that ICOS can inhibit the phagocytosis of Dilox-LDL by HASMCs and down-regulate the expression of lipid phagocytic receptor CD36 on HASMCs, which might contribute to the anti-AS effect of ICOS.

ICOS is an important costimulatory molecule on T cells. Binding to its ligand, ICOSL, plays an important role in $\mathrm{T}$ cell proliferation and activation, maintenance of $\mathrm{T}$ help (Th) cell response, and participating in the $\mathrm{CD}^{+} \mathrm{T}$ cellular immune response. There have been numerous studies on ICOS in tumor immunotherapy, and a multiple studies have found that ICOS participates in a variety of tumor disease processes and plays a dual role in tumor occurrence 
and immune response (25). With the increasing public attention to immune costimulatory molecules, research into ICOS in AS has gradually increased in recent years. Down-regulation of ICOS expression has been detected in aortic atherosclerotic plaques of $\mathrm{ApoE}^{-/-}$rats (20). High-fatfed ICOS gene deficient $\mathrm{ApoE}^{-/-}$mice had a $77 \%$ increase in early atherosclerotic plaque, and a $36 \%$ increase in late atherosclerotic plaque (19). ICOS gene deletion enhances the response of mouse $\mathrm{T}$ cells to hyperlipidemia and increases AS (26). In this study, we further revealed that the mechanism by which ICOS exerts its protective effect against AS may be through down-regulating the expression of the lipid phagocytic receptor, CD36, to inhibit the lipid phagocytosis of HASMCs.

Based on the fact that the muscle-derived foam cells in AS are derived from smooth muscle cells, we added a large amount of ox-LDL to HASMCs to construct an in vitro cellular AS model. Excessive lipid uptake is the main reason for the formation of lipid accumulation. The lipid uptake in HASMCs after ox-LDL treatment was significantly increased; however, after stimulation with JK-ICOS cells or rICOS protein, this phenomenon was markedly reduced. This indicates that ICOS can prevent ox-LDL-induced lipid accumulation by reducing the uptake of ox-LDL.

It is well established that CD36 is one of the main proteins involved in the progression of AS (23). CD36 stimulates the proliferation of vascular smooth muscle cells (VSMCs) by up-regulating the expression of cyclin $\mathrm{A}$, as well as promoting neointimal hyperplasia, collagen deposition, and the development of obstructive vascular diseases (11). In this study, experiments were performed to detect the expression of CD36 by RT-qPCR, western blotting, and immunofluorescence staining to determine whether the related molecules of ICOS inhibition of lipid accumulation are directly related to CD36. We found that the mRNA and protein expression levels of CD36 in HASMCs co-cultured with JK-ICOS cells under ox-LDL stimulation decreased. The immunofluorescence staining results showed that co-culture with JK-ICOS cells could significantly down-regulate the expression of CD36 in HASMCs induced by ox-LDL. However, there was no difference in the CD36 expression between the ox-LDLtreated group and the ox-LDL- and JK-EV-treated group, which suggested that overexpressed ICOS gene could downregulate the expression of CD36 through T cells.

Subsequently, in order to discover whether the effect of JK-ICOS cells in inhibiting CD36 expression is through the $\mathrm{T}$ cell itself or through the ICOS gene, we used rICOS protein instead of JK-ICOS cells to process HASMCs. The results showed that the mRNA and protein expression levels of CD36 on HASMCs under rICOS protein stimulation also exhibited a downward trend, yet the degree of decline was not as obvious as that of the JK-ICOS cell co-culture. Immunofluorescence staining showed identical results. This suggests that the inhibitory effect of JK-ICOS cells on the expression of CD36 on HASMCs may be mainly achieved by affecting JurKat cells, and the effect of ICOS gene alone is not a decisive factor. Unfortunately, we have not detected the protein and function of JurKat cells, after ICOS overexpression, and not able to verify the downstream signaling pathway of ICOS affecting CD36, which requires further research in the future.

As early as 2013, researchers have summarized the signaling pathways involved in AS, and PPAR $\gamma, \mathrm{Nrf2}$, and TLR4/Syk signaling pathways are all involved (27). The oxLDL-PPAR $\gamma$-CD36 axis can promote lipid accumulation in human umbilical vein smooth muscle cells. Downregulation of PPAR $\gamma$ prevents ox-LDL-induced lipid accumulation and inhibits the expression of CD36 (14). Knockout of Nrf2 can reduce cholesterol influx by downregulating the expression of $\mathrm{CD} 36$, resulting in a $53 \%$ reduction in the degree of AS in $\mathrm{Nrf2}^{-/-} \mathrm{ApoE}^{-/-}$mice (28). These studies demonstrate that $\mathrm{CD} 36$ and its related signal pathways play a crucial role in the development mechanism of AS. However, studies have also shown that some signaling pathways, such as cyclic adenosine monophosphate (cAMP)/ exchange protein directly activated by cAMP (cAMP/ Epac1) signaling, can express anti-AS through the LOX-1 receptor, which regulates the uptake of ox-LDL, but has no effect on the expression of CD36 (29). The results of our study suggest that ICOS can down-regulate the expression of CD36, but its specific mechanism has not been further studied. Therefore, it is necessary to conduct further experiments to explore the mechanisms through which ICOS affects the specific signal pathway of CD36.

\section{Conclusions}

Our results revealed that one of the mechanisms of ICOS inhibition of lipid phagocytosis and accumulation of HASMCs is by inhibiting the expression of phagocytic receptor, CD36. Its participation in the anti-AS effect may be related to the inhibition of ox-LDL-induced myogenic foam cell formation. 


\section{Acknowledgments}

The authors thank their lab members Qunji Zhang, Linkai Tan, Qiaoting Deng, Xiaodong Gu and Ruiqiang Weng for technical help and comments, as well as Dr. $\mathrm{Xin} \mathrm{Mu}$ at Tianjin University for helpful discussion. The authors would like to thank Professor Mu Xin of Tianjin University for participating in the preliminary conception of the research ideas and experimental design, and Qunji Zhang and Linkai Tan of Center for Precision Medicine, Meizhou People's Hospital (Huangtang Hospital), Meizhou Academy of Medical Sciences, for participating in the research work of experimental design and guidance. Also, the authors thank other colleagues (who were not listed in the authorship) from the Center for Precision Medicine, Meizhou People's Hospital (Huangtang Hospital), Meizhou Academy of Medical Sciences for their helpful comments on the manuscript.

Funding: This study was supported by the Natural Science Foundation of Guangdong Province (2020A1515010430), the Guangdong Provincial Key Laboratory of Precision Medicine and Clinical Translation Research of Hakka Population (2018B030322003), and the Science and Technology Program of Meizhou (2019B0202001).

\section{Footnote}

Reporting Checklist: The authors have completed the MDAR reporting checklist. Available at https://jtd.amegroups.com/ article/view/10.21037/jtd-21-1901/rc

Data Sharing Statement: Available at https://jtd.amegroups. com/article/view/10.21037/jtd-21-1901/dss

Conflicts of Interest: All authors have completed the ICMJE uniform disclosure form (available at https://jtd.amegroups. com/article/view/10.21037/jtd-21-1901/coif). The authors have no conflicts of interest to declare.

Ethical Statement: The authors are accountable for all aspects of the work in ensuring that questions related to the accuracy or integrity of any part of the work are appropriately investigated and resolved.

Open Access Statement: This is an Open Access article distributed in accordance with the Creative Commons Attribution-NonCommercial-NoDerivs 4.0 International License (CC BY-NC-ND 4.0), which permits the non- commercial replication and distribution of the article with the strict proviso that no changes or edits are made and the original work is properly cited (including links to both the formal publication through the relevant DOI and the license). See: https://creativecommons.org/licenses/by-nc-nd/4.0/.

\section{References}

1. Wang Y, Xie Y, Zhang A, et al. Exosomes: An emerging factor in atherosclerosis. Biomed Pharmacother 2019;115:108951.

2. Wigren M, Rattik S, Yao Mattisson I, et al. Lack of Ability to Present Antigens on Major Histocompatibility Complex Class II Molecules Aggravates Atherosclerosis in ApoE-/Mice. Circulation 2019;139:2554-66.

3. Bai Y, Liu X, Chen Q, et al. Myricetin ameliorates oxLDL-induced HUVECs apoptosis and inflammation via lncRNA GAS5 upregulating the expression of miR-29a3p. Sci Rep 2021;11:19637.

4. Sun L, Gai J, Shi S, et al. Protease-Activated Receptor 2 (PAR-2) Antagonist AZ3451 Mitigates Oxidized LowDensity Lipoprotein (Ox-LDL)-Induced Damage and Endothelial Inflammation. Chem Res Toxicol 2021;34:2202-8.

5. Rinne $P$, Rami $M$, Nuutinen $S$, et al. Melanocortin 1 Receptor Signaling Regulates Cholesterol Transport in Macrophages. Circulation 2017;136:83-97.

6. Huang L, Chambliss KL, Gao X, et al. SR-B1 drives endothelial cell LDL transcytosis via DOCK4 to promote atherosclerosis. Nature 2019;569:565-9.

7. Runge MS, Stouffer GA, Patterson C. Netter's Cardiology. Netter's Cardiology; 2015.

8. Ross R. The pathogenesis of atherosclerosis: a perspective for the 1990s. Nature 1993;362:801-9.

9. Yang M, Silverstein RL. CD36 signaling in vascular redox stress. Free Radic Biol Med 2019;136:159-71.

10. Płóciennikowska A, Hromada-Judycka A, Borzęcka K, et al. Co-operation of TLR4 and raft proteins in LPSinduced pro-inflammatory signaling. Cell Mol Life Sci 2015;72:557-81.

11. Yue H, Febbraio M, Klenotic PA, et al. CD36 Enhances Vascular Smooth Muscle Cell Proliferation and Development of Neointimal Hyperplasia. Arterioscler Thromb Vasc Biol 2019;39:263-75.

12. Yazgan B, Sozen E, Karademir B, et al. CD36 expression in peripheral blood mononuclear cells reflects the onset of atherosclerosis. Biofactors 2018;44:588-96.

13. Li X, Zhang T, Geng J, et al. Advanced Oxidation Protein 
Products Promote Lipotoxicity and Tubulointerstitial Fibrosis via CD36/ $\beta$-Catenin Pathway in Diabetic Nephropathy. Antioxid Redox Signal 2019;31:521-38.

14. Zhuang JL, Liu YY, Li ZZ, et al. Amentoflavone prevents ox-LDL-induced lipid accumulation by suppressing the PPAR $\gamma / C D 36$ signal pathway. Toxicol Appl Pharmacol 2021;431:115733.

15. Dutta RK, Lee JN, Maharjan Y, et al. Catalase deficiency facilitates the shuttling of free fatty acid to brown adipose tissue through lipolysis mediated by ROS during sustained fasting. Cell Biosci 2021;11:201.

16. Riera-Heredia N, Lutfi E, Balbuena-Pecino S, et al. The autophagy response during adipogenesis of primary cultured rainbow trout (Oncorhynchus mykiss) adipocytes. Comp Biochem Physiol B Biochem Mol Biol 2022;258:110700.

17. Wikenheiser DJ, Stumhofer JS. ICOS Co-Stimulation: Friend or Foe? Front Immunol 2016;7:304.

18. Mittelsteadt KL, Hayes ET, Campbell DJ. ICOS signaling limits regulatory $\mathrm{T}$ cell accumulation and function in visceral adipose tissue. J Exp Med 2021;218:e20201142.

19. Afek A, Harats D, Roth A, et al. A functional role for inducible costimulator (ICOS) in atherosclerosis. Atherosclerosis 2005;183:57-63.

20. Zhong Z, Zhang Q, Tan L, et al. T cell co-stimulator inducible co-stimulatory (ICOS) exerts potential antiatherosclerotic roles through downregulation of vascular smooth muscle phagocytosis and proliferation. Ann Transl Med 2020;8:1597.

21. Choromańska B, Myśliwiec P, Choromańska K, et al. The role of CD36 receptor in the pathogenesis of atherosclerosis. Adv Clin Exp Med 2017;26:717-22.

Cite this article as: Liang $\mathrm{M}$, Guo $\mathrm{X}, \mathrm{Wu} \mathrm{H}$, Zhong Z. Inducible co-stimulator inhibits lipid phagocytosis of human aortic smooth muscle cells by down-regulating CD36 expression. J Thorac Dis 2022;14(1):147-157. doi: 10.21037/jtd21-1901
22. Cao X, Zhu N, Li L, et al. Y-box binding protein 1 regulates ox-LDL mediated inflammatory responses and lipid uptake in macrophages. Free Radic Biol Med 2019;141:10-20.

23. Tian K, Xu Y, Sahebkar A, et al. CD36 in Atherosclerosis: Pathophysiological Mechanisms and Therapeutic Implications. Curr Atheroscler Rep 2020;22:59.

24. Song Q, Hu Z, Xie X, et al. Zafirlukast prevented oxLDL-induced formation of foam cells. Toxicol Appl Pharmacol 2020;409:115295.

25. Amatore F, Gorvel L, Olive D. Role of Inducible CoStimulator (ICOS) in cancer immunotherapy. Expert Opin Biol Ther 2020;20:141-50.

26. Gotsman I, Grabie N, Gupta R, et al. Impaired regulatory $\mathrm{T}$-cell response and enhanced atherosclerosis in the absence of inducible costimulatory molecule. Circulation 2006;114:2047-55.

27. Hopkins PN. Molecular biology of atherosclerosis. Physiol Rev 2013;93:1317-542.

28. Barajas B, Che N, Yin F, et al. NF-E2-related factor 2 promotes atherosclerosis by effects on plasma lipoproteins and cholesterol transport that overshadow antioxidant protection. Arterioscler Thromb Vasc Biol 2011;31:58-66.

29. Robichaux WG 3rd, Mei FC, Yang W, et al. Epac1 (Exchange Protein Directly Activated by cAMP 1) Upregulates LOX-1 (Oxidized Low-Density Lipoprotein Receptor 1) to Promote Foam Cell Formation and Atherosclerosis Development. Arterioscler Thromb Vasc Biol 2020;40:e322-35.

(English Language Editor: A. Kassem) 